\title{
Quality of life measures (EORTC QLQ-C30 and SF-36) as predictors of survival in palliative colorectal and lung cancer patients
}

\author{
GUNN E. GRANDE, B.A. (HONs.), M.Sc., M.PHIL., PH.D., ${ }^{1}$ MORAG C. FARQUHAR, B.SC. (HONs.), M.SC., PH.D., ${ }^{2}$ \\ STEPHEN I.G. BARCLAY, M.A., B.M., B.CH., M.SC., F.R.C.G.P., M.D., ${ }^{2}$ AND \\ CHRISTOPHER J. TODD, B.A., M.A., PH.D., C.PSYCHOL., A.F.B.PS.S. ${ }^{1}$ \\ ${ }^{1}$ School of Nursing, Midwifery \& Social Work, The University of Manchester, Manchester, United Kingdom \\ ${ }^{2}$ General Practice \& Primary Care Research Unit, University of Manchester, Institute of Public Health, Manchester, \\ United Kingdom
}

(RECEIVED June 26, 2008; ACCEPTED July 19, 2008)

\begin{abstract}
Objective: Self-reported health-related quality of life (HRQoL) is an important predictor of survival alongside clinical variables and physicians' prediction. This study assessed whether better prediction is achieved using generic (SF-36) HRQoL measures or cancer-specific (EORTC QLQ-C30) measures that include symptoms.

Method: Fifty-four lung and 46 colorectal patients comprised the sample. Ninety-four died before study conclusion. EORTC QLQ-C30 and SF-36 scores and demographic and clinical information were collected at baseline. Follow-up was 5 years. Deaths were flagged by the Office of National Statistics. Cox regression survival analyses were conducted. Surviving cases were censored in the analysis.

Results: Univariate analyses showed that survival was significantly associated with better EORTC QLQ-C30 physical functioning, role functioning, and global health and less dyspnea and appetite loss. For the SF-36, survival was significantly associated with better emotional role functioning, general health, energy/vitality, and social functioning. The SF-36 summary score for mental health was significantly related to better survival, whereas the SF-36 summary score for physical health was not. In the multivariate analysis, only the SF-36 mental health summary score remained an independent, significant predictor, mainly due to considerable intercorrelations between HRQoL scales. However, models combining the SF-36 mental health summary score with diagnosis explained a similar amount of variance $(12 \%-13 \%)$ as models combining diagnosis with single scale SF-36 Energy/Vitality or EORTC QLQ-C30 Appetite Loss.

Significance of results: HRQoL contributes significantly to prediction of survival. Generic measures are at least as useful as disease-specific measures including symptoms. Intercorrelations between HRQoL variables and between HRQoL and clinical variables makes it difficult to identify prime predictors. We need to identify variables that are as independent of each other as possible to maximize predictive power and produce more consistent results.
\end{abstract}

KEYWORDS: Palliative care, Quality of life, Survival, Colorectal neoplasms, Lung neoplasms

Address correspondence and reprint requests to: Gunn E. Grande, School of Nursing, Midwifery and Social Work, The University of Manchester, Jean McFarlane Building, Oxford Road, Manchester M13 9PL, United Kingdom. E-mail: gunn. grande@manchester.ac.uk

\section{INTRODUCTION}

The prediction of survival is described as one of the most important issues in palliative clinical practice and research (Maltoni \& Tassinari, 2004). It plays an important role in the provision of information to 
patients and their families, patient management, discharge planning, assessment of patients for social security benefits, and establishment of patients' eligibility to participate in clinical trials (den Daas, 1995; Vigano et al., 2000). However, clinical prediction in advanced cancer has repeatedly been shown to be unreliable (den Daas, 1995; Oxenham \& Cornbleet, 1998; Glare et al., 2003).

The main variables that have been investigated as predictors of survival are clinical factors, including biological data and clinicians' assessment of the patient's status, clinicians' prediction of survival (which is likely to take clinical variables into account), and patients' self assessments of their health-related quality of life (HRQoL), including symptom burden.

Literature reviews have clearly demonstrated the value of clinical variables (Vigano et al., 2000) and clinicians' estimates (Glare et al., 2003) in the prediction of survival. However, a large number of studies suggest that patients' self-assessed HRQoL makes an important and independent contribution to prediction of survival alongside clinical variables and professional assessment for patients with lung cancer (Langendijk et al., 2000; Montazeri et al., 2001; Dharma-Wardene et al., 2004; Efficace et al., 2006), advanced colorectal cancer (Maisey et al., 2002; Efficace et al., 2005; Lis et al., 2006), advanced cancer in general (Coates et al., 1997; Dancey et al., 1997), and the terminally ill (Tamburini et al., 1996; Llobera et al., 2000). In contrast, a few studies have concluded that the contribution of self-assessed variables was limited once clinical variables were accounted for in samples of lung cancer (Herndon et al., 1999) and terminal cancer patients (Toscani et al., 2005), respectively.

If self-reported HRQoL aids prediction of survival, we should aim to establish what type of measure is most useful in adding predictive information alongside clinical measures and clinicians' predictions. The EORTC QLQ-C30 (Herndon et al., 1999) is an extensively used cancer-specific measure that has been designed to capture patients' functional status in several domains (physical, psychological, and social), their global health status/quality of life (QoL), and symptom severity. It has been investigated extensively in the prediction of cancer survival. Several studies have found its global health status/QoL scale to be the strongest HRQoL predictor in lung cancer patients (Langendijk et al., 2000; Montazeri et al., 2001), advanced colorectal cancer patients (Maisey et al., 2002), and in advanced cancer patients in general (Coates et al., 1997; Dancey et al., 1997). However, a few studies have found EORTC QLQ-C30 symptom scores (Herndon et al., 1999; Efficace et al., 2006) or social functioning (Efficace et al.,
2005) to be more important. Nevertheless, it appears that patients' general well-being may overall be more predictive of survival than symptom severity or functional status.

It seems plausible that patients' ratings of their general health and QoL should contribute more to prediction alongside clinical variables than self-rated symptoms or functional status, as the latter to some extent can be assessed by others, although not perfectly. Although clinicians are reasonably able to assess observable symptoms, they are poor at assessing patients' QoL (Fowlie et al., 1989; Regan et al., 1991). However, if HRQoL measures make their greatest contributions to prediction of survival by measuring global health/QoL, a more comprehensive generic measure may add more predictive value than cancer-specific measures that include symptoms, particularly in situations where clinical information is already fairly comprehensive.

This study, therefore, aimed to test whether a generic measure of HRQoL, the SF-36 (Ware \& Sherbourne, 1992) was an equally good or better predictor of survival than the cancer-specific EORTC QLQ-C30 in a sample of colorectal and lung cancer patients who were defined as needing palliative care. The mental component summary score (MCS) of the SF-36 has been reported to predict survival in lung cancer patients alongside another generic quality of life measure (Manser et al., 2006). The SF-36 was tested together with the EORTC QLQC30 in a study by Camilleri-Brennan and Steele (2001) that suggested that EORTC QLQ-C30 subscales provided better prediction. However, the study sample was rectal cancer patients undergoing surgery, and $88 \%$ were alive at the end of the study. The present study therefore constitutes the first comparison of the performance of the generic SF-36 versus the cancer-specific EORTC QLQ-C30 in predicting survival in cancer patients defined as needing palliative care.

\section{METHODS}

\section{Sample}

The sample consisted of patients with colorectal or lung cancer, which represent the most common cancer diagnoses encompassing both men and women. Patients were identified through consecutive discharge summaries and outpatient consultation letters from participating oncology consultants and surgeons at a UK Hospital National Health Service Trust. Inclusion criteria were age 16 years or above, diagnosis of lung or colorectal cancer, and explicit reference to the need for palliation in the hospital notes and/or a disease staging deemed by a clinician as 
giving clear indication of palliative status (e.g., liver metastases for colorectal cancer).

\section{Procedure}

Data were collected as part of a longitudinal study investigating patients' and carers' needs and service use. General practitioners (GPs) of eligible patients were asked by letter whether the patient could be contacted for the study (Farquhar et al., 2002). Patients deemed contactable by the GP were sent a study introduction letter with a reply slip.

Those who agreed to participate were interviewed at home for the general study. After the interview, the SF-36, the EORTC QLQ-C30, and a reply envelope were left with the patient with a request to complete the questionnaires "within the next couple of days." If respondents requested, interviewers provided help with questionnaire completion. The sample was flagged for deaths by the Office of National Statistics and followed for 5 years from the baseline interview. Stage of colorectal cancer and type of lung cancer were identified from patients' medical notes. Approval was obtained from the Cambridge Local Research Ethics Committee.

\section{Measures}

The EORTC QLQ-C30 (EORTC Study Group, 1995) is a HRQoL measure specific to cancer, whereas the SF-36 (Ware \& Sherbourne, 1992) is a generic measure. Table 1 shows the individual EORTC QLQ-C30 and SF-36 scales (excluding the EORTC QLQ-C30 Financial Difficulties scale, which is unlikely to relate to prognosis). The SF-36 has been subjected to principal components analysis yielding two

Table 1. Age, sex, and baseline HRQoL scores by cancer diagnosis

\begin{tabular}{|c|c|c|c|}
\hline & Colorectal & Lung & $\begin{array}{l}\text { Test statistic } \\
\text { and } p \text { value }\end{array}$ \\
\hline Mean age $(S D)$ & $71.5(9.5)$ & $69.2(9.0)$ & $\begin{array}{c}t=1.263, d f=98 \\
p=.210\end{array}$ \\
\hline \multirow[t]{2}{*}{ No. of males } & $29(63.0 \%)$ & $33(61.1 \%)$ & $\begin{array}{c}\chi^{2}=0.039, d f=1 \\
p=.843\end{array}$ \\
\hline & $\begin{array}{l}\text { Colorectal median } \\
\text { scores (IQR) }\end{array}$ & $\begin{array}{l}\text { Lung median } \\
\text { scores (IQR) }\end{array}$ & \\
\hline \multicolumn{4}{|l|}{ EORTC QLQ-C30 dimensions } \\
\hline Physical functioning & $60.0(40.0)$ & $40.0(40.0)$ & $z=2.198(p=.028)$ \\
\hline Role functioning & $66.7(50.0)$ & $33.3(83.3)$ & $z=1.562(p=.118)$ \\
\hline Cognitive functioning & $83.3(33.3)$ & $83.3(33.3)$ & $z=0.506(p=.613)$ \\
\hline Emotional functioning & $83.3(18.8)$ & $79.2(39.6)$ & $z=1.339(p=.180)$ \\
\hline Social functioning & $66.7(66.7)$ & $66.7(66.7)$ & $z=0.571(p=.568)$ \\
\hline Global health status/QoL & $50.0(25.0)$ & $50.0(41.7)$ & $z=1.388(p=.165)$ \\
\hline Fatigue & $44.4(33.3)$ & $55.6(44.4)$ & $z=1.530(p=.126)$ \\
\hline Nausea/vomiting & $0.0(16.7)$ & $0.0(16.7)$ & $z=0.678(p=.498)$ \\
\hline Pain & $33.3(50.0)$ & $16.7(50.0)$ & $z=0.256(p=.798)$ \\
\hline Dyspnea & $0.0(33.3)$ & $33.3(66.7)$ & $z=3.684(p=.0002)$ \\
\hline Insomnia & $33.3(33.3)$ & $33.3(75.0)$ & $z=1.902(p=.057)$ \\
\hline Appetite loss & $0.0(33.3)$ & $33.3(66.7)$ & $z=1.648(p=.099)$ \\
\hline Constipation & $0.0(33.3)$ & $0.0(33.3)$ & $z=0.461(p=.645)$ \\
\hline Diarrhea & $0.0(33.3)$ & $0.0(0.0)$ & $z=2.006(p=.044)$ \\
\hline \multicolumn{4}{|l|}{ SF-36 dimensions } \\
\hline Physical functioning & $40.0(35.0)$ & $30.0(55.0)$ & $z=1.441(p=.150)$ \\
\hline Role-Physical ${ }^{\mathrm{a}}$ & $0.0(43.7)$ & $0.0(25.0)$ & $z=0.611(p=.541)$ \\
\hline Role-Emotional ${ }^{\mathrm{b}}$ & $100.0(100.0)$ & $33.3(100.0)$ & $z=1.954(p=.051)$ \\
\hline Bodily pain & $55.6(66.7)$ & $55.6(55.6)$ & $z=0.747(p=.455)$ \\
\hline General health & $50.0(28.3)$ & $35.0(32.0)$ & $z=2.410(p=.016)$ \\
\hline Energy/vitality & $45.0(30.0)$ & $35.0(41.3)$ & $z=1.812(p=.070)$ \\
\hline Social function & $55.6(72.2)$ & $27.8(66.7)$ & $z=1.599(p=.110)$ \\
\hline Mental Health & $78.0(29.0)$ & $72.0(24.0)$ & $z=0.927(p=.354)$ \\
\hline Physical component summary (PCS) & $22.2(16.6)$ & $21.6(16.3)$ & $z=0.859(p=.390)$ \\
\hline Mental component summary (MCS) & $52.1(21.3)$ & $39.6(22.8)$ & $z=1.934(p=.053)$ \\
\hline No. of respondents HRQoL scores & $42-43$ & $48-50$ & \\
\hline
\end{tabular}

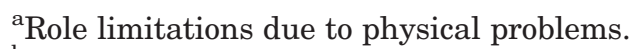

${ }^{b}$ Role limitations due to emotional problems. 
summary measures: one for responses representing a physical component summary (PCS) and one for responses representing a mental component summary (MCS; Jenkinson et al., 1997).

\section{Analysis}

Demographic and clinical differences between diagnostic groups at baseline were tested using chisquare and $t$ tests. HRQoL differences were tested using nonparametric Mann-Whitney $U$ tests and correlations calculated using Spearman's correlation coefficient $(R \mathrm{~s})$ due to considerable skew or kurtosis in many of the HRQoL variables (Siegel \& Castellan, 1988).

Relationships between baseline variables and survival were investigated through univariate Cox regression analyses. To assess appropriateness for this analysis, the proportional hazards assumption was tested for each variable by assessing whether the Kaplan-Meier survival curves for variable categories were parallel (continuous variable categories were created by dividing the variable at the median; Campbell, 2001).

HRQoL variables associated with survival at $p<.1$ in the univariate analyses (Hosmer \& Lemeshow, 2000) were entered into multivariate Cox regression analyses. Separate multivariate analyses were conducted for SF-36 and for EORTC QLQ-C30 variables. Multivariate analyses were first performed using the enter method to assess the resulting model with all identified variables entered, and second using a forward stepwise method whereby the most significant variable is entered into the model first, to assess which variables appeared most important in predicting survival. Significant HRQoL variables were finally tested alongside any significant demographic and clinical variables. Procedures ensured that there were more than 10 cases per variable within any multivariate analysis. SPSS version 13.0 was used, and the variance explained by the resulting models was calculated according to Nagelkerke (1991).

\section{RESULTS}

\section{Patient Sample}

Of 242 patients identified, 28 died before contact, 14 were deemed "unsuitable" by their GP, 69 refused, and 31 gave no response. This yielded 54 lung and 46 colorectal cancer patients who took part in the study. Fifty lung and 43 colorectal cancer patients completed the EORTC QLQ-C30 and the SF-36 at baseline. Five years after the baseline interview, 94 patients had died. Surviving cases were censored in the analysis ( 5 colorectal and 1 lung).

Table 1 shows the baseline sample characteristics. Compared to lung cancer patients, colorectal cancer patients had better EORTC QLQ-C30 physical functioning and lower levels of dyspnea. Lung cancer patients had lower levels of diarrhea, although low prevalence yielded a median of 0 in both groups.

\section{Univariate Cox Regression Analysis}

All variables conformed to the proportional hazards assumption, except the EORTC QLQ-C30 Diarrhea scale.

Survival from baseline was considerably skewed, and median and quartile values for days of survival are reported. Colorectal cancer patients had significantly better overall survival from baseline, that is, reduced risk of death, compared to lung cancer patients. However, it was patients with Dukes C, rather than Dukes D, that experienced better survival relative to lung cancer (Table 2). Survival was not significantly longer for non-small-cell lung cancer (243 [81-323]) than small-cell lung cancer (228 [51-276]). There were no age and sex differences.

For the EORTC QLQ-C30 (Table 3), high baseline scores (better functioning) on Physical Functioning, Role Functioning and Global Health/QoL were significantly associated with reduced risk of death, whereas high scores (worse symptoms) on Dyspnea and Appetite Loss were related to worse survival. For the SF-36, high baseline scores (better functioning) on Role-Emotional, General Health, Energy/

Table 2. Univariate Cox regression analyses of diagnosis, disease stage, and survival

\begin{tabular}{lccr}
\hline \hline & $\begin{array}{c}\text { Median (quartiles) survival } \\
\text { from baseline (days) }\end{array}$ & $B(S E)$ & $\operatorname{Exp}(B)(95 \% \mathrm{CI})$ \\
\hline Diagnosis & $334(143-866)$ & $-0.58(0.22)$ & $0.56(0.37-0.86)$ \\
$\quad$ Colorectal $(n=46)$ & $245(91-478)$ & 0 & .01 \\
Lung $(n=54)$ & & & $.0 .47(0.26-0.84)$ \\
Disease stage & $334(107-1822)$ & $-0.74(0.30)$ & .01 \\
Dukes C $(n=19)$ & $307(135-704)$ & $-0.37(0.27)$ & $0.69(0.41-1.17)$ \\
Dukes D $(n=21)$ & $245(91-478)$ & 0 & 1 \\
Lung $(n=54)$ & & & .17 \\
\end{tabular}


Table 3. Univariate Cox regression models: Individual HRQoL scales and survival

\begin{tabular}{|c|c|c|c|c|}
\hline & $n$ & $B(S E)$ & $\operatorname{Exp}(B)(95 \%$ CI $)$ & $P$ \\
\hline \multicolumn{5}{|l|}{ EORTC QLQ C-30 } \\
\hline Physical functioning & 93 & $-0.008(0.004)$ & $0.992(0.984-1.000)$ & .042 \\
\hline Role functioning & 93 & $-0.008(0.003)$ & $0.992(0.986-0.998)$ & .012 \\
\hline Emotional functioning & 90 & $-0.002(0.004)$ & $0.998(0.989-1.006)$ & .583 \\
\hline Cognitive functioning & 90 & $0.002(0.004)$ & $1.002(0.994-1.010)$ & .562 \\
\hline Social functioning & 90 & $-0.004(0.003)$ & $0.996(0.990-1.001)$ & .139 \\
\hline Global health/QoL & 90 & $-0.012(0.004)$ & $0.988(0.981-0.996)$ & .004 \\
\hline Fatigue & 93 & $0.007(0.004)$ & $1.007(1.000-1.014)$ & .057 \\
\hline Nausea/vomiting & 93 & $0.006(0.005)$ & $1.006(0.996-1.016)$ & .257 \\
\hline Pain & 93 & $0.006(0.004)$ & $1.006(0.998-1.013)$ & .148 \\
\hline Dyspnea & 93 & $0.007(0.003)$ & $1.007(1.001-1.013)$ & .020 \\
\hline Insomnia & 93 & $0.000(0.003)$ & $1.000(0.994-0.005)$ & .881 \\
\hline Appetite loss & 93 & $0.008(0.003)$ & $1.008(1.002-1.014)$ & .014 \\
\hline Constipation & 90 & $0.000(0.004)$ & $1.000(0.992-0.007)$ & .923 \\
\hline Diarrhea & 90 & $0.004(0.006)$ & $1.004(0.993-1.015)$ & .477 \\
\hline \multicolumn{5}{|l|}{ SF-36 } \\
\hline Physical functioning & 93 & $-0.004(0.004)$ & $0.996(0.988-1.003)$ & .285 \\
\hline Role-Physical ${ }^{\mathrm{a}}$ & 86 & $-0.004(0.003)$ & $0.996(0.989-1.002)$ & .196 \\
\hline Role-Emotional ${ }^{\mathrm{b}}$ & 87 & $-0.006(0.002)$ & $0.994(0.989-0.999)$ & .011 \\
\hline Bodily pain & 93 & $-0.006(0.004)$ & $0.994(0.988-1.001)$ & .103 \\
\hline General health & 89 & $-0.017(0.005)$ & $0.983(0.973-0.994)$ & .002 \\
\hline Energy/vitality & 92 & $-0.011(0.005)$ & $0.989(0.979-0.999)$ & .026 \\
\hline Social functioning & 91 & $-0.007(0.003)$ & $0.993(0.987-0.999)$ & .022 \\
\hline Mental health & 91 & $-0.008(0.006)$ & $0.991(0.979-1.003)$ & .159 \\
\hline Physical component summary (PCS) & 77 & $-0.018(0.010)$ & $0.982(0.963-1.002)$ & .073 \\
\hline Mental component summary (MCS) & 77 & $-0.027(0.010)$ & $0.973(0.955-0.992)$ & .006 \\
\hline
\end{tabular}

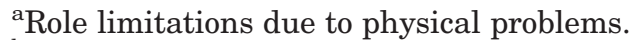

${ }^{\mathrm{b}}$ Role limitations due to emotional problems.

Vitality, and Social Functioning were significantly associated with improved survival. A high mental summary score (MCS) was significantly related to better survival, whereas a high physical summary score (PCS) was not.

\section{Multivariate Cox Regression Analysis}

EORTC QLQ-C30 variables related to survival at $p<.1$ in the univariate analysis were all significantly intercorrelated ( $R$ s range from .356 to .793$)$; likewise, SF-36 individual scales $(R \mathrm{~s}$ range from .534 to .784$)$ and PCS and MCS summary scales $(R \mathrm{~s}=.331, p=.003)$ were significantly intercorrelated.

When the six EORTC QLQ-C30 variables at $p<.1$ in the univariate analysis were entered into a multivariate Cox regression model simultaneously, none of the variables showed a significant relationship with survival, that is, no variable remained significant when all the other variables were taken into account. When employing forward stepwise regression, whereby the most significant variable is entered into the model first, Appetite Loss emerged as a significant predictor. Once Appetite Loss was controlled for, none of the other variables remained significant. However, its selection was based on marginal differences in statistical significance between highly intercorrelated variables. Therefore this result is likely to be a function of this particular statistical analysis, rather than demonstrating the importance of loss of appetite relative to other prognostic variables. Prior to entry of Appetite Loss into the model, Global Health/QoL showed a similarly strong relationship with survival in the multivariate analysis.

Likewise, when the six SF-36 individual scales at $p<.01$ were entered simultaneously into a multivariate model, none of the variables remained a significant predictor of survival. When employing forward stepwise regression, Energy/Vitality emerged as a significant predictor. Again none of the other variables remained significant when this variable was controlled for. However, the selection of Energy/Vitality was again based on marginal statistical differences, for example, with General Health, with which it was strongly correlated. Therefore, this result is again likely to be a function of this statistical analysis and data set.

In contrast, when the SF-36 PCS and MCS were entered into a multivariate model simultaneously, the relationship between the MCS and survival remained significant when physical aspects were controlled for (Table 4). 
Table 4. Multivariate Cox regression model $(\mathrm{n}=77)$

\begin{tabular}{lccr}
\hline \hline SF-36 summary score & $B(\mathrm{SE})$ & $\operatorname{Exp}(B)(95 \% \mathrm{CI})$ & $p$ \\
\hline Mental component summary & $-0.024(0.011)$ & $0.976(0.955-0.998)$ & $R^{2}$ \\
Physical component summary & $-0.007(0.010)$ & $0.993(0.973-1.014)$ & .029 \\
\end{tabular}

As lung and colorectal cancer patients differed in some of their HRQoL scores, any relationship between such variables and survival may be due to the difference in survival between diagnoses. Significant HRQoL variables were therefore entered into a multivariate Cox regression together with disease stage and diagnosis. Appetite Loss, Energy/Vitality, and MCS all remained significant predictors when disease stage and diagnosis were controlled for (Table 5). Disease stage and diagnosis were no longer significant when MCS was taken into account, although adding stage and diagnosis to MCS increased the variance explained (Tables 4 and 5). The amount of variance accounted for by each model in Table 5 was very similar, regardless of type of HRQoL variable considered $(12 \%-13 \%)$.

\section{DISCUSSION}

\section{Summary}

Colorectal cancer patients had better survival than lung cancer patients, with Dukes $\mathrm{C}$ stage rather than Dukes D stage colorectal cancer representing the better survival over lung cancer. For the EORTC QLQ-C30, both the general health and functioning scales (Physical Functioning, Role Functioning, and Global Health/QoL) and symptom scales (Dyspnea and Appetite Loss) significantly predicted survival. These findings correspond with Vigano et al.'s (2000) review of clinical factors and survival where (clinician assessed) functional status, dyspnea, and anorexia emerged as being important, although this review considered cancer patients with median survival of 3 months or less. For the SF-36, Role-Emotional, General Health, Energy/Vitality, and Social Functioning were significantly associated with survival. A high SF-36 MCS was significantly related to better survival whereas, perhaps surprisingly, a high PCS was not.

In the multivariate analysis of HRQoL scales, none of the single scales emerged as a clear independent survival predictor due to considerable intercorrelation between scales. Although forward stepwise regression analysis made the statistical procedure select one variable for each instrument (Appetite Loss for EORTC QLQ-C30 and Energy/Vitality for SF-36), their selection may well be a function of this particular analysis, as other variables were nearly equally strong contenders. This included the EORTC QLQ-C30 Global Health/QoL variable, which has been found to be a strong predictor in previous research (Dancey et al., 1997; Montazeri et al., 2001; Maisey et al., 2002).

Table 5. Multivariate Cox regression models with main HRQoL variables and diagnosis

\begin{tabular}{llll}
\hline \hline & \multicolumn{1}{c}{$B(\mathrm{SE})$} & $\operatorname{Exp}(B)(95 \% \mathrm{CI})$ & $p$ \\
\hline Model 1 $(n=89)$ & & & $R^{2}$ \\
$\quad$ EORTC appetite loss & $0.007(0.003)$ & $1.007(1.001-1.013)$ & .029 \\
$\quad$ Disease stage & $-0.683(0.308)$ & $0.505(0.276-0.924)$ & .083 \\
$\quad$ Dukes C & $-0.249(0.274)$ & $0.780(0.456-1.334)$ & .027 \\
$\quad$ Dukes D & 0 & 1 & .364 \\
$\quad$ Lung & $-0.011(0.005)$ & $0.989(0.979-0.999)$ & .027 \\
Model 2 $(n=88)$ & $-0.820(0.314)$ & $0.441(0.238-0.816)$ & .032 \\
SF-36 energy/vitality & $-0.278(0.273)$ & $0.758(0.444-1.292)$ & .009 \\
Disease stage & 0 & 1 & .308 \\
$\quad$ Dukes C & & & .13 \\
$\quad$ Dukes D & $-0.025(0.11)$ & $0.976(0.956-0.996)$ & .019 \\
$\quad$ Lung & $-0.572(0.325)$ & $0.565(0.298-1.068)$ & .211 \\
Model 3 $(n=75)$ & $-0.121(0.312)$ & $0.886(0.481-1.632)$ & .079 \\
SF-36 MCS & 0 & 1 & .697 \\
Disease stage & & & .13 \\
$\quad$ Dukes C & & & \\
$\quad$ Dukes D & & & \\
$\quad$ Lung & & & \\
\hline \hline
\end{tabular}


In contrast to the single scales, the SF-36 MCS scale retained a clear relationship with survival even when physical HRQoL aspects (PCS) were controlled for. However, when combined with disease stage and diagnosis, the MCS performed no better than Appetite Loss (a single-item symptom measure) or Energy/Vitality (one of the scales contributing to the MCS score) in the amount of variance in survival explained. Thus although the HRQoL variables contributed something over and beyond basic clinical variables, it remains unclear which HRQoL variable was the better predictor and whether the generic or specific HRQoL measure performed better.

\section{Limitations}

A limitation of the study may be the representativeness of the sample. Patients were identified through oncology clinics. Many died before contact, the most ill may have been "selected out" by their GPs, and palliative care patients who participate in research probably have longer survival than those who refuse (Grande et al., 1997). The results may therefore represent patients with advanced cancer rather than more end-stage palliative patients. The most important predictive variables for these patients may be quite different from those toward the end of life. Nevertheless, the independent contribution of selfreported HRQoL has been demonstrated in previous studies of patients with a median survival of 56-59 days (Tamburini et al., 1996; Llobera et al., 2000).

A second study limitation was the inability to test for clinical variables beyond diagnosis. More comprehensive clinical measures may again have given more prominence to clinical variables in the survival equation. However, most studies into HRQoL included a more comprehensive range of clinical variables and still concluded that HRQoL was an important contributor to survival (Tamburini et al., 1996; Coates et al., 1997; Langendijk et al., 2000; Llobera et al., 2000; Montazeri et al., 2001; Maisey et al., 2002; Dharma-Wardene et al., 2004; Efficace et al., 2005, 2006; Lis et al., 2006).

Finally, the variance explained in survival was small, possibly due to the limited range of variables incorporated, but larger studies with more comprehensive measurement of clinical variables may still leave over $70 \%$ of variance in survival unexplained (Toscani et al., 2005).

\section{Implications}

The study limitations clearly point to the need for larger studies with more representative samples, a more comprehensive range of variables, and repeated measurements to assess the predictive importance of variables at different stages. However, the study results also highlight a more fundamental methodological point that needs to be addressed if this field is to progress. The high degree of intercorrelation between HRQoL single scales made it difficult to identify one as the better predictor. Other research confirms the high intercorrelations between subscales of HRQoL measures (Coates et al., 1997). Furthermore, HRQoL scales correlate considerably with clinical factors such as performance status and weight loss (Herndon et al., 1999). The high degree of intercorrelation between variables may yield apparently conflicting results between studies into survival that really only represent small differences in how individual multivariate statistical analyses have worked out. Intercorrelation also means there is considerable redundancy in the information used to predict survival.

This suggests a need to develop and/or utilize variables that are as independent of each other as possible to maximize predictive power and produce more consistent results. The development of the SF36 mental and physical composite scales (MCS and PCS) may represent one such way forward. These were developed from the single SF-36 scales through factor analysis with orthogonal rotation to be as unrelated to each other as possible (Jenkinson et al., 1997) and correspondingly showed the lowest intercorrelations of the predictor variables. The MCS scales therefore represent a psychological component that is as "uncontaminated" by physical aspects as possible and vice versa. In our analysis the MCS retained a significant relationship with survival even when entered alongside physical components (PCS) and alongside diagnosis/stage, whereas diagnosis/ stage was no longer significant alongside MCS. Although this specific result may reflect the early palliative stage of this particular patient sample and/or the limited range of clinical variables, the desirability of identifying clear, independent predictor variables remains.

Maltoni and Tassinari (2004) call for systematic composite evaluation combining clinicians' survival estimates with performance status, symptoms, and biomedical data through prognostic score instruments as a means of improving prediction and resolving conflicting findings. To this we would add the need to include self-reported HRQoL, where possible, to improve predictive power. However, HRQoL variables need to be systematically investigated to assess where they provide the clearest added, independent contribution. Their value may, for instance, lie in measurement of more general well-being, which may reflect underlying disease that cannot fully be detected through tests or clinician assessment (Maisey et al., 2002). We would also add the need to assess all of the component variables of prognostic 
instruments, both clinical and HRQoL, for their independence of other components and their unique contribution in order to design instruments with maximum predictive power.

In studies to date a large amount of variance in survival remains unexplained (Toscani et al., 2005), and a more systematic, comprehensive approach is needed to improve this. Although we are probably still unlikely to accurately predict survival for individuals, improved prediction of probabilities can nevertheless provide valuable guidance to clinicians in the difficult art of outlining and planning future options with patients and their families.

\section{Conclusions}

Our study confirms that self-reported HRQoL is significantly and independently associated with survival in palliative patients. It also suggests that a generic mental health measure may be an equally good, possibly at times better, predictor of survival compared to self-reported physical health for patients relatively early in their disease trajectory. However, considerable intercorrelations between HRQoL measures yielded unclear results. This suggests that to improve prediction there is a general need to invest in improved variable selection and design to produce a complementary and comprehensive set of independent predictor variables, as well as a need for larger scale studies with representative samples and repeated measurement.

\section{ACKNOWLEDGMENTS}

The authors thank all those who contributed to and participated in this study: consultants and hospital administrative staff for recruitment assistance, all participating patients and carers, Malcolm Campbell for statistical advice, and Anna Martin for assistance with recruitment and data collection. The study was funded by the NHS R\&D Primary/Secondary Interface Programme and the Directorate of Health and Social Care Midlands and East of England grant RCC11095.

\section{REFERENCES}

Camilleri-Brennan, J. \& Steele, R.J.C. (2001). Prospective analysis of quality of life and survival following mesorectal excision for rectal cancer. British Journal of Surgery, 88, 1617-1622.

Campbell, M.J. (2001). Statistics at Square Two: Understanding Modern Statistical Applications in Medicine. London: BMJ Books.

Coates, A., Porzsolt, F. \& Osoba, D. (1997). Quality of life in oncology practice: Prognostic value of EORTC QLQ-C30 scores in patients with advanced malignancy. European Journal of Cancer, 33, 1025-1030.

Dancey, J., Zee, B., Osoba, D., et al. (1997). Quality of life scores: An independent prognostic variable in a general population of cancer patients receiving chemotherapy. Quality of Life Research, 6, 151-158.

den Daas, N. (1995). Estimating length of survival in endstage cancer: A review of the literature. Journal of Pain and Symptom Management, 10, 548-555.

Dharma-Wardene, M., Au, H.J., Hanson, J., et al. (2004). Baseline FACT-G score is a predictor of survival for advanced lung cancer. Quality of Life Research, 13, 1209-1216.

Efficace, F., Bottomley, A., Coens, C., et al. (2005). Does a patient's self-reported health-related quality of life predict survival beyond key biomedical data in advanced colorectal cancer? European Journal of Cancer, 42, 42-49.

Efficace, F., Bottomley, A., Smit, E.F., et al. (2006). Is a patient's self-reported health-related quality of life a prognostic factor for survival in non-small-cell lung cancer patients? A multivariate analysis of prognostic factors of EORTC study 08975. Annals of Oncology, 17, 1698-704.

EORTC Study Group on Quality of Life. (1995). EORTC Scoring Manual. Brussels: EORTC.

Farquhar, M., Grande, G., Todd, C., et al. (2002). Defining patients as palliative: Hospital doctors' versus general practitioners' perceptions. Palliative Medicine, 16, $247-250$.

Fowlie, M., Berkeley, J. \& Dingwall-Fordyce, I. (1989). Quality of life in advanced cancer: The benefits of asking the patient. Palliative Medicine, 3, 55-59.

Glare, P., Virik, K., Jones, M., et al. (2003). A systematic review of physicians' survival predictions in terminally ill cancer patients. British Medical Journal, 327, 195-201.

Grande, G.E., Todd, C.J. \& Barclay, S.I.G. (1997). Support needs in the last year of life: Patient and carer dilemmas. Palliative Medicine, 11, 202-208.

Herndon, J.E., Fleishman, S., Kornblith, A.B., et al. (1999). Is quality of life predictive of the survival of patients with advanced nonsmall cell lung carcinoma? Cancer, $85,333-340$.

Hosmer, D.H. \& Lemeshow, S. (2000). Applied Logistic Regression, 2nd ed. New York: Wiley.

Jenkinson, C., Layte, R. \& Lawrence, K. (1997). Development and testing of the SF-36 summary scale scores in the United Kingdom: Results from a large scale survey and clinical trial. Medical Care, 35, 410-416.

Langendijk, H., Aaronson, N.K., de Jong, J.M., et al. (2000). The prognostic impact of quality of life assessed with the EORTC QLQ-C30 in inoperable non-small cell lung carcinoma treated with radiotherapy. Radiotherapy \& Oncology, 55, 19-25.

Lis, C.G., Gupta, D., Granick, J., et al. (2006). Can patient satisfaction with quality of life predict survival in advanced colorectal cancer? Supportive Care in Cancer, $14,1104-1110$.

Llobera, J., Esteva, M., Rifa, J., et al. (2000). Terminal cancer, duration and prediction of survival time. European Journal of Cancer, 36, 2036-2043.

Maisey, N.R., Norman, A., Watson, M., et al. (2002). Baseline quality of life predicts survival in patients with advanced colorectal cancer. European Journal of Cancer, 38, 1351-1357.

Maltoni, M. \& Tassinari, D. (2004). Prognostic assessment in terminally ill cancer patients: From evidence-based knowledge to a patient-physician relationship and back. Palliative Medicine, 18, 77-79.

Manser, R.L., Wright, G., Byrnes, G., et al. (2006). Validity of the Assessment of Quality of Life (AQoL) utility instrument in patients with operable and inoperable lung cancer. Lung Cancer, 53, 217-229. 
Montazeri, A., Milroy, R., Hole, D., et al. (2001). Quality of life in lung cancer patients, as an important prognostic factor. Lung Cancer, 31, 233-240.

Nagelkerke, N.J.D. (1991). A note on a general definition of the coefficient of determination. Biometrika, 78, 691-692.

Oxenham, D. \& Cornbleet, M.A. (1998). Accuracy of prediction of survival by different professional groups in a hospice. Palliative Medicine, 12, 117-118.

Regan, J., Yarnold, J., Jones, P.W., et al. (1991). Palliation and life quality in lung cancer: How good are clinicians at judging treatment outcome? British Journal of Cancer, 64, 396-400.

Siegel, S. \& Castellan, N.J. (1988). Non-parametric Statistics for the Behavioural Sciences, 2nd ed. Singapore: McGraw-Hill.
Tamburini, M., Brunelli, C., Rosso, S., et al. (1996). Prognostic value of quality of life scores in terminal cancer patients. Journal of Pain and Symptom Management, 11, 32-41.

Toscani, F., Brunelli, C., Miccinesi, G., et al. (2005). Predicting survival in terminal cancer patients: Clinical observation or quality-of-life evaluation? Palliative Medicine, $19,220-227$.

Vigano, A., Dorgan, M., Buckingham, J., et al. (2000). Survival prediction in terminal cancer patients: A systematic review of the medical literature. Palliative Medicine, $14,363-374$.

Ware, J.E. \& Sherbourne, C.D. (1992). The MOS 36-item Short Form Health Survey (SF-36), 1. Conceptual framework and item selection. Medical Care, 30, 473-483. 